\title{
Dual-composite right-left-handed transmission lines for the design of compact diplexers
}

\section{$\begin{array}{lll}\text { V. González-Posadas } & \text { J.L. Jiménez-Martín } & \\ & \text { A. Parra-Cerrada } & 1\end{array}$}

L.E. García-Muñoz $z^{2} \quad$ D. Segovia-Vargas ${ }^{2}$

${ }^{1}$ Department of Ingenieria Audiovisual y Comunicaciones, Universidad Politécnica de Madrid, Ctra de Valencia $\mathrm{km} 7$, 28031, Madrid, Spain

${ }^{2}$ Department of Signal Theory and Communications, Carlos III University in Madrid, Avenida de la Universidad 30, 29811. Leganés, Madrid, Spain

E-mail: dani@tsc.uc3m.es

Abstract: In this study, the use of dual-composite right-left-handed (D-CRLH) transmission lines is proposed for the design of diplexers. The D-CRLH diplexers present advantages over conventional diplexers such as smaller size (especially in the ultra high frequency band). The design procedure and the design equations are presented in this paper. The non-linear phase response of a D-CRLH transmission line has been used to design diplexers at any arbitrary frequencies. Finally, two diplexers have been designed and manufactured: one for quite near frequencies and the other for further ones. The simulation results show good agreement with the measurements. A sensitivity analysis has been provided to show the robustness of this kind of circuits.

\section{Introduction}

Diplexers have been widely used since the early 1960 s [1] as a means to separate two different output frequencies from one common input signal. With the growth of the wireless services, the need of compact and costly efficient diplexers is growing on and on. Recently, in [2], a generalised treatment to the design of diplexers loaded with any complex load has been given. In addition, the current trend comes from the technological need to design compact diplexer circuits. Traditionally, microwave diplexers have been realised in either distributed- or lumped-element technology. Transmission line or distributed technology presents an inherent difficulty to achieve compact circuits. The use of ring resonators [3] has been proposed as a possible way to make filters compact. However, the extension to compact diplexers is not direct.

New technologies are being proposed in order to achieve compactness in diplexers. One of them is the multilayered low-temperature cofired ceramics (LTCC) $[4,5]$. The results that this multilayered technology is offering are quite promising. On the other side, the compositeright-left-handed (CRLH) technology has emerged as a new technology offering new potentials, mainly, on multifrequency and on miniaturisation $[6,7]$. The realisation of diplexers with CRLH lines has not shown a great development till now. Different topologies to build CRLH diplexers have been used. In this way, in [8] splitring-resonators were used in a classical diplexer topology.

In an attempt to achieve a compact CRLH diplexer, a multilayered three-dimensional diplexer was proposed in [9]. Compactness was achieved at a price of large losses and a relative important frequency shift. Other original planar (but rather complex and non-compact) topology for diplexer designs has been proposed in [10]. Recently, in [11] a diplexer based on non-conventional dual-mode rat race coupler has been proposed. Its main drawback is its non-compactness, especially when frequencies are low. In addition, its design cannot be extended to general multiplexer realisations (more than two output ports). 
Then, it seems that conventional CRLH lines present different problems when applying to diplexer designs.

Recently, in $[12,13]$ a modification of the conventional $\mathrm{CRLH}$ transmission called dual-CRLH (D-CRLH) has been proposed. This structure presents a dual equivalent circuit in comparison with the CRLH transmission line and has a series parallel and a shunt series LC tank circuits. This topological duality results in dual properties. The D-CRLH indeed exhibits its left-handed band at high frequencies and its right-handed band at low frequencies. Besides, it is of stop-band nature and pass-band in opposition to the CRLH which is low-frequency lefthanded and high-frequency right-handed. This structure has been seldom used for microwave circuit design, that is $[14,15]$.

This paper presents a general design strategy, based on D-CRLH transmission lines, to obtain compact diplexers working at any arbitrary pair of frequencies. Its design parameters are the cut-off frequencies and the number of sections for the D-CRLH lines or the insertion losses. Most designs make use of two sections, although this number will mainly be determined by the separation between frequencies and the allowed insertion losses. The analysis has shown that the response is kept over a larger bandwidth than the corresponding prototype with conventional CRLH lines and with lower losses in the corresponding pass-bands. In addition, two prototypes (one working at near frequencies and other at further ones) have been manufactured and measured. Finally, a sensitivity analysis has been undertaken to prove the prototype robustness.

\section{D-CRLH diplexer principles}

\section{$2.1 D$-CRLH lines principles}

D-CRLH structures have been firstly proposed by Caloz [13], where it is posed that the structure in Fig. 1 behaves as the complementary structure of the conventional CRLH cell. This structure has a series parallel and a shunt series LC tank circuits. The D-CRLH indeed exhibits its lefthanded band at high frequencies and its right-handed band at low frequencies and is of stop-band nature.

The equations running the performance of the $\mathrm{D}-\mathrm{CRLH}$ balanced line are given in (1)-(3). If the resonance

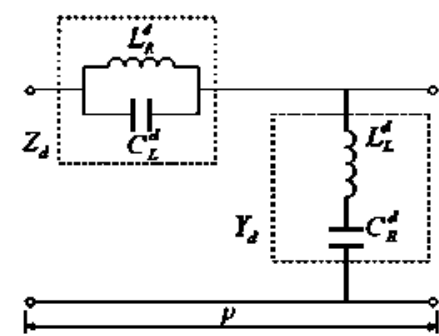

Figure 1 Schematic of the D-CRLH transmission line frequencies of the shunt LC tank and the series LC tank are $\omega_{\mathrm{sh}}$ and $\omega_{\mathrm{se}}$ a balanced condition can be achieved by satisfying (1)

$$
\omega_{\mathrm{sh}}=\sqrt{\frac{1}{L_{\mathrm{L}} C_{\mathrm{R}}}}=\omega_{\mathrm{se}}=\sqrt{\frac{1}{L_{\mathrm{R}} C_{\mathrm{L}}}}
$$

Once the D-CRLH line is balanced, let us define a righthanded frequency and a left-handed frequency as

$$
\omega_{\mathrm{R}}=\sqrt{\frac{1}{L_{\mathrm{R}} C_{\mathrm{R}}}}, \quad \omega_{\mathrm{L}}=\sqrt{\frac{1}{L_{\mathrm{L}} C_{\mathrm{L}}}}
$$

And the condition defined in (1) can be rewritten as

$$
\omega_{0}=\sqrt{\omega_{\mathrm{sh}} \omega_{\mathrm{se}}}=\sqrt{\omega_{\mathrm{L}} \omega_{\mathrm{R}}}=\omega_{\mathrm{sh}}=\omega_{\mathrm{se}}
$$

The characteristic impedance of the line is particularised for the long wavelength region and balanced condition as follows

$$
\begin{array}{r}
Z_{0}(\omega)=\sqrt{\frac{Z_{\mathrm{D}-\mathrm{CRLH}}}{Y_{\mathrm{D}-\mathrm{CRLH}}}}=Z_{\mathrm{R}} \sqrt{\frac{1-\left(\omega / \omega_{\mathrm{sh}}\right)^{2}}{1-\left(\omega / \omega_{\mathrm{se}}\right)^{2}}} \\
{ }_{\omega_{\text {se }}=\omega_{\text {sh }}} Z_{0}=Z_{\mathrm{R}}=Z_{\mathrm{L}}=\sqrt{\frac{L_{\mathrm{R}}}{C_{\mathrm{R}}}}=\sqrt{\frac{L_{\mathrm{L}}}{C_{\mathrm{L}}}}
\end{array}
$$

where $Z_{\mathrm{D}-\mathrm{CRLH}}$ and $Y_{\mathrm{D}-\mathrm{CRLH}}$ are the series impedance and the shunt admittance of the D-CRLH section. Finally, the propagation constant per unit cell ( $p$ ) for the balance case is given as

$$
\begin{aligned}
\eta(\omega) & =\sqrt{Z_{\mathrm{D}-\mathrm{CRLH}} Y_{\mathrm{D}-\mathrm{CRLH}}}=\sqrt{\mathrm{j} \frac{\omega L_{\mathrm{R}}}{1-\left(\omega / \omega_{\mathrm{se}}\right)^{2}} \mathrm{j} \frac{\omega C_{\mathrm{R}}}{1-\left(\omega / \omega_{\mathrm{sh}}\right)^{2}}} \\
& =\mathrm{j} \frac{\omega}{\omega_{\mathrm{R}}} \sqrt{\frac{\omega_{\mathrm{se}}^{2} \omega_{\mathrm{sh}}^{2}}{\omega_{\mathrm{se}}^{2} \omega_{\mathrm{sh}}^{2}-\omega_{\mathrm{se}}^{2} \omega^{2}-\omega_{\mathrm{sh}}^{2} \omega^{2}+\omega^{4}}} \\
& =\mathrm{j} \frac{\omega \omega_{\mathrm{L}}}{\sqrt{\omega_{\mathrm{se}}^{2} \omega_{\mathrm{sh}}^{2}-\omega^{2}\left(\omega_{\mathrm{se}}^{2}+\omega_{\mathrm{sh}}^{2}\right)+\omega^{4}}} \\
& =\mathrm{j} \frac{\omega \omega_{\mathrm{L}}}{\left.(\omega)^{2}-\omega_{0}^{2}\right)}=\mathrm{j} \beta(\omega)
\end{aligned}
$$

The unit cell of Fig. $\mathbf{1}$ is repeated to form a periodic infinite transmission line. The input impedance is recursively repeated at the successive nodes. Then, the overall input impedance is given as

$$
\begin{aligned}
Z_{\text {in }}= & Z_{\mathrm{D}-\mathrm{CRLH}}+\left[\frac{1}{Y_{\mathrm{D}-\mathrm{CRLH}}} / / Z_{\text {in }}\right]=Z_{\mathrm{D}-\mathrm{CRLH}} \\
& +\frac{\left(Z_{\text {in }} / Y_{\mathrm{D}-\mathrm{CRLH}}\right)}{\left(1 / Y_{\mathrm{D}-\mathrm{CRLH}}\right)+Z_{\text {in }}} \Rightarrow Z_{\text {in }} \\
= & \frac{Z_{\mathrm{D}-\mathrm{CRLH}}}{2}\left[1 \pm \sqrt{1+\frac{4}{Z_{\mathrm{D}-\mathrm{CRLH}} Y_{\mathrm{D}-\mathrm{CRLH}}}}\right]
\end{aligned}
$$


This infinite transmission line approach can be taken to calculate the cut-off frequencies by applying the procedure presented in [7] for CRLH lines to D-CRLH lines. That approach provides accurate results for the $-10 \mathrm{~dB}$ cut-off frequency when the number of cells is larger than 3 . Then, by substituting $Z_{\mathrm{D}-\mathrm{CRLH}}$ and $Y_{\mathrm{D}-\mathrm{CRLH}}$ with the expressions in terms of $\omega_{\mathrm{sh}}$ and $\omega_{\mathrm{se}}$ and calculating the roots of the radicand of $Z_{\text {in }}$ in (6) results in the following cut-off frequencies for the left- and right-handed regions

$$
\omega_{\mathrm{CL} / \mathrm{R}}=\omega_{0} \sqrt{1+\frac{\omega_{\mathrm{L}}}{8 \omega_{\mathrm{R}}} \pm \sqrt{\frac{\omega_{\mathrm{L}}}{4 \omega_{\mathrm{R}}} \sqrt{1+\frac{\omega_{\mathrm{L}}}{16 \omega_{\mathrm{R}}}}}}
$$

where the sign ' + ' is for the left-handed high-pass cut-off and the sign '-' is for the right-handed low-pass cut-off. It must be noted that $\omega_{\mathrm{CL}}>\omega_{\mathrm{CR}}$.

Figs. 2 and 3 show the performance of a dual-CRLH line. The amplitude and phase of the $s_{21}$ parameter have been represented. It can be seen that there is a band-stop at $970 \mathrm{MHz}$ instead of the passing one that there would be for the conventional CRLH case at the desired working frequency. In addition, the phase of the $s_{21}$ gives us the

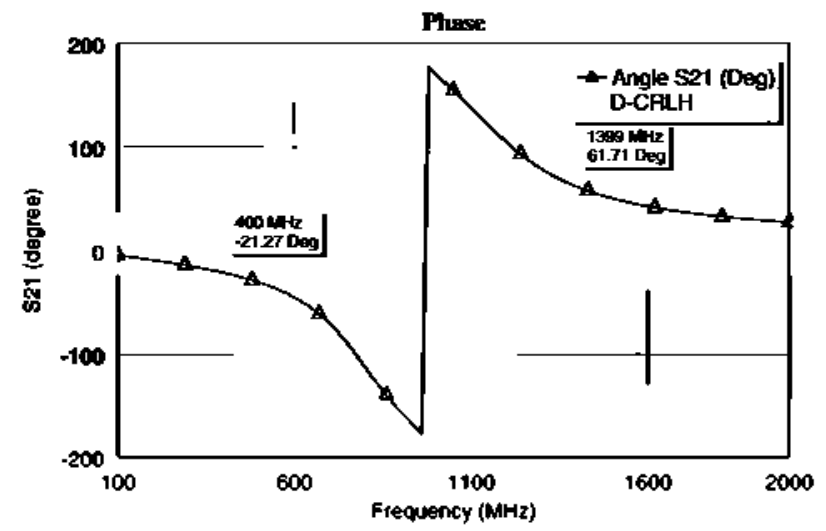

Figure $2 D$-CRLH phase response

The parameter are $L_{\mathrm{R}}=6 \mathrm{nH}, C_{\mathrm{R}}=2.4 \mathrm{pF}, L_{\mathrm{L}}=11.13 \mathrm{nH}$ and $c_{\mathrm{L}}=\mathbf{4 . 4 5 2 \mathrm { pF }}$

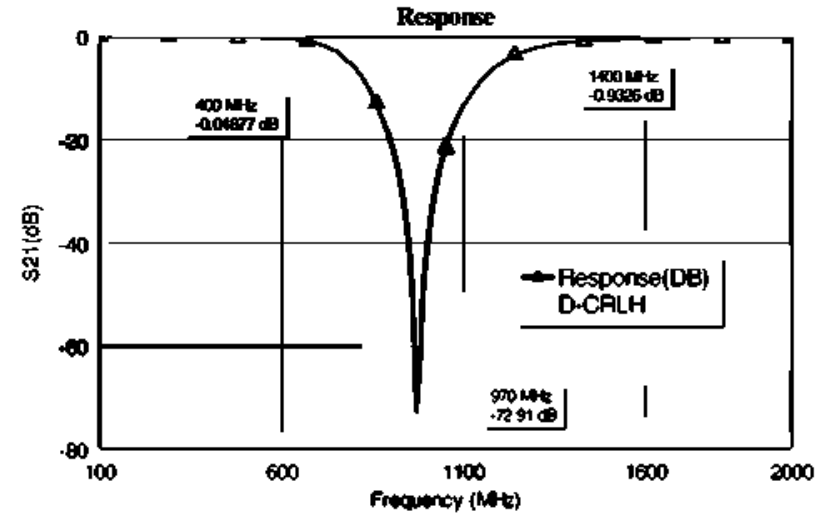

Figure 3 D-CRLH amplitude response

The parameter are $L_{R}=6 \mathrm{nH}, C_{R}=2.4 \mathrm{pF}, L_{L}=11.13 \mathrm{nH}$ and $c_{\mathrm{L}}=4.452 \mathrm{pF}$ information about the left- or right-handed performance of the D-CRLH line. The phase of $s_{21}$ is given by $-\beta d$ (where $d$ is the length of the transmission line). For lefthanded region, the phase of $s_{21}$ would be positive since $\beta$ is negative, while for the right-handed region, the phase of $s_{21}$ would be negative since $\beta$ is positive. Then, for the proposed D-CRLH transmission structure, the line is right-handed for frequencies lower than $970 \mathrm{MH} z$, while it is left-handed for frequencies higher than $970 \mathrm{MHz}$.

\subsection{Diplexer design principles}

The design of a diplexer based on CRLH lines has shown two drawbacks, one coming from the losses at the pass bands and the other one from the fact that near frequencies cannot be properly diplexed. As a diplexer can be based either on allowing some frequency pass-bands or on rejecting the frequency stop-bands, the use of D-CRLH transmission lines can help to overcome the previous problems by working with the complementary rejection frequency bands. Thus, the band-stop performance of a D-CRLH line is the key to design a diplexer.

A diplexer separating the lower frequency $\left(f_{\text {lower }}\right)$ from the higher one $\left(f_{\text {higher }}\right)$ has the first D-CRLH line at the upper branch with a band-stop at $f_{\text {higher }}$ and a pass band at $f_{\text {lower }}$, while the second D-CRLH line at the bottom branch presents a band-stop at $f_{\text {lower }}$ and a pass band at $f_{\text {higher }}$. Then, according to Fig. 4, the design frequency of the upper branch, $f_{0}$, will be $f_{\text {higher }}$ (to allow $f_{\text {lower }}$ pass at port 2) and $f_{\text {lower }}$ for the bottom branch (to allow fhigher pass at port 3). According to the performance of the D-CRLH line in Fig. 3, it can be said that the D-CRLH line in the upper branch of the diplexer is right-handed at $f_{\text {lower, }}$, while the D-CRLH line in the bottom branch of the diplexer is left-handed at $f_{\text {higher, }}$ just the opposite as for a conventional CRLH transmission line.

In the proposed D-CRLH diplexer design, it is essential to have right-handed propagation at the passing frequency, flower, of the upper branch, while left-handed propagation at the second passing frequency, $f_{\text {higher, }}$ from the bottom branch. In order to assure that, let us introduce a factor $k(N)$ as the ratio between the band-stop frequency and the right-handed frequency, $f_{\mathrm{r}}$, for right-handed propagation or the opposite for left-handed propagation, that is, the ratio between the left-handed frequency, $f$, and the bandstop frequency. This factor assures that we have the correct performance of the corresponding D-CRLH

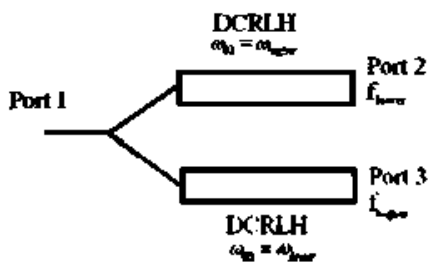

Figure 4 D-CRLH proposed diplexer structure 
line: left-handed performance towards the right side of the desired band-stop frequency and right-handed performance towards the left side of the band-stop frequency. This factor depends on the number of cells in the D-CRLH line and on the frequency separation. Without loss of generality, let us assume that the number of cells is 1 for the following design procedure:

- Choose the band-stop frequencies for the two balanced D-CRLH lines in any of the diplexer branches. These frequencies are chosen so that the band-stop frequency of the upper branch allows the lower frequency pass, while the band-stop frequency of the bottom branch allows the higher frequency pass through this branch. Then, if the upper branch is denoted by sub-index 2 , while the bottom branch is denoted by sub-index 3 , we have the following frequency assignment

$$
\omega_{02}=\omega_{\text {higher }}, \quad \omega_{03}=\omega_{\text {lower }}, \quad \omega_{02}>\omega_{03}
$$

- Define the right-handed frequency of the D-CRLH section of the upper branch, $\omega_{2 \mathrm{R}}$, and the left-handed frequency of the D-CRLH section of the bottom branch, $\omega_{3 L}$, as follows

$$
\omega_{3 \mathrm{~L}}=\omega_{\text {lower }} k(N), \quad \omega_{2 \mathrm{R}}=\frac{\omega_{\text {higher }}}{k(N)}
$$

- For symmetry, we can define the left-handed frequency of the D-CRLH section of the upper branch, $\omega_{2 L}$, and the right-handed frequency of the D-CRLH section of the bottom branch, $\omega_{3 \mathrm{R}}$ as follows

$$
\omega_{3 \mathrm{R}}=\frac{\omega_{\text {lower }}}{k(N)}, \quad \omega_{2 \mathrm{~L}}=\omega_{\text {higher }} k(N)
$$

- Introduce the values of the right- and left-handed frequencies for the branch 2 and the branch 3 in (7) to obtain the cut-off frequencies (in order to generalise the expression $\omega_{\text {hower }}$ or $\omega_{\text {higher }}$ have been changed by $\omega_{0}(11)$ ) (see (11))
- Finally, in order that the diplexer can properly separate the two frequencies, it has been chosen that the right-handed cut-off frequency of the upper branch be at the right side of the left-handed cut-off frequency of the bottom branch. This means that $\omega_{\mathrm{C} 2 \mathrm{R}}>\omega_{\mathrm{C} 3 \mathrm{~L}}$. If this is introduced in (11) it leads to the following transcendent equation (see (12))

If the ratio between the higher and lower frequency is called $R \quad\left(R=\omega_{\text {highel }} / \omega_{\text {lower }}\right)$ then, the limit for the previous inequality will be given by the following equation

$\left(R^{2}-1\right)+\left(R^{2}-1\right) \frac{[k(N)]^{2}}{8}-\left(R^{2}+1\right) \frac{[k(N)]}{2} \sqrt{1+\frac{[k(N)]^{2}}{16}}=0$

Equation (13) is a transcendent equation that is represented in Fig. 5. The $k$ factor can be approached by a second-, third- or fourth-order polynomial without making an important error. For the third-order case, the expression of

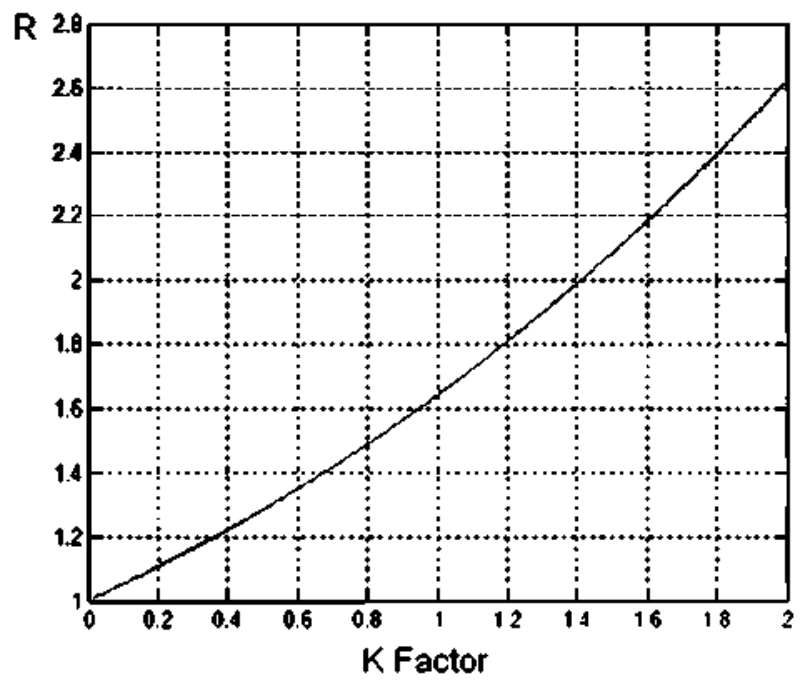

Figure $5 K$ factor as a function of the ratio $R$

$$
\begin{aligned}
\omega_{\mathrm{CL} / \mathrm{R}} & =\omega_{0} \sqrt{1+\frac{\omega_{o} k(N)}{8\left(\omega_{o} / k(N)\right)} \pm \sqrt{\frac{\omega_{0} k(N)}{4\left(\omega_{0} / k(N)\right)} \sqrt{1+\frac{\omega_{o} k(N)}{16\left(\omega_{o} / k(N)\right)}}}} \\
& =\omega_{0} \sqrt{1+\frac{[k(N)]^{2}}{8} \pm \sqrt{\frac{[k(N)]^{2}}{4} \sqrt{1+\frac{[k(N)]^{2}}{16}}}}
\end{aligned}
$$

$$
\omega_{\text {lower }}<\omega_{\text {higher }} \frac{\sqrt{1+\left([k(N)]^{2} / 8\right)-\sqrt{\left([k(N)]^{2} / 4\right) \sqrt{1+\left([k(N)]^{2} / 16\right)}}}}{\sqrt{1+\left([k(N)]^{2} / 8\right)+\sqrt{\left([k(N)]^{2} / 4\right) \sqrt{1+\left([k(N)]^{2} / 16\right)}}}}
$$


the third-order polynomial is given as

$$
k(N)=0.1309 R^{3}-1.0143 R^{2}+3.5343 R-2.6442
$$

Once the value of the $k$ factor and of the frequency ratio $R$ has been determined, the values for each branch left-handed and right-handed capacitors can be found out. Thus for the branch rejecting the higher frequency and allowing the lower one (port 2 in Fig. 4), the values for the components are given as (sub-index of the component is taken as the number of the output port)

$$
\begin{gathered}
L_{2 \mathrm{R}}=\frac{Z_{0} k(N)}{\omega_{\text {ligher }}}, \quad L_{2 \mathrm{~L}}=\frac{Z_{0}}{\omega_{\text {higher }} k(N)} \\
C_{2 \mathrm{~L}}=\frac{L_{2 \mathrm{~L}}}{Z_{0}^{2}}, \quad C_{2 \mathrm{R}}=\frac{L_{2 \mathrm{R}}}{Z_{0}^{2}}
\end{gathered}
$$

And for the branch rejecting the lower frequency and allowing the higher one (port 3 in Fig. 4), the values for the components are given as (sub-index of the component is taken as the number of the output port)

$$
\begin{gathered}
L_{3 \mathrm{R}}=\frac{Z_{0} k(N)}{\omega_{\text {lower }}}, \quad L_{3 \mathrm{~L}}=\frac{Z_{0}}{\omega_{\text {lower }} k(N)} \\
C_{3 \mathrm{~L}}=\frac{L_{3 \mathrm{~L}}}{Z_{0}^{2}}, \quad C_{3 \mathrm{R}}=\frac{L_{3 \mathrm{R}}}{Z_{0}^{2}}
\end{gathered}
$$

Equations (15) and (16) provide the overall inductances and capacitances for the D-CRLH lines when they only have one section. When the number of sections is increased and as all the sections of the D-CRLH transmission line are identical, the values for the corresponding inductances and capacitances of any D-CRLH section are obtained as follows

$$
\begin{array}{ll}
L_{2,3 \mathrm{R}, \mathrm{N}}=\frac{L_{2,3 \mathrm{R}}}{N}, & L_{2, \hat{\mathrm{L}}, \mathrm{N}}=L_{2,3 \mathrm{~L}} N \\
C_{2,3 \mathrm{R}, \mathrm{N}}=\frac{C_{2,3 \mathrm{R}}}{N}, & C_{2,3 \mathrm{~L}, \mathrm{~N}}=C_{2,3 \mathrm{~L}} N
\end{array}
$$

A discussion on the number of sections and on the $\mathrm{D}$-CRLH performance can be done now. It must be emphasised that, as the goal is to design a D-CRLH transmission line based on its elementary sections, all the sections are equal. This is opposite to what it happens when conventional filter theory is applied where the sections are in general different. For the present case, the objective is to design the so-called D-CRLH transmission line with all the sections identical in order to have right-handed propagation at lower frequencies and left-handed propagation at higher frequencies. In [13], the D-CRLH performance has been proven for a 10-cell transmission line structure. If the number of cells is reduced, the left-handed performance still maintains as it has been shown in [16]. The only dependence on the number of cells comes from the fact that the slope of the response between the low-frequency right-handed region and the high-frequency left-handed one is more abrupt when the number of cells is larger. However, from the diplexer design point of view, a tradeoff between the desired losses and the rejection factor at the non-desired frequency in the corresponding branch must be considered. In this way, for most single designs low order ( 1 or 2$)$ for the identical D-CRLH sections are enough to achieve the desired performance.

\section{Experimental results}

Two diplexers have been designed (with AWR), manufactured and measured to prove the previous design procedure. Since one of the most important features is its miniaturisation, both designs are applied to the low microwave frequency band: first a diplexer for separate TETRA band from GSM band and, secondly, a diplexer for separating close frequencies, that is GSM from GALILEO bands. It has to be pointed out that the sensitivity of the second diplexer is much larger. Then, a sensitivity analysis will be finally provided in order to see the design robustness.

\subsection{Diplexer for separate frequencies}

A TETRA-GSM diplexer has been designed and measured at 380 and $960 \mathrm{MHz}$. Following the design considerations in (9)-(14) the corresponding component values are shown in Table 1. This table shows the designed values and the commercial chosen. In addition, the tolerance and the selfresonance frequency of each component are also shown to see the circuit sensitivity. Sub-index two corresponds to the branch rejecting the higher frequency $(960 \mathrm{MHz})$ and allowing the lower one $(380 \mathrm{MHz})$, while sub-index three corresponds to the branch rejecting the lower frequency $(380 \mathrm{MHz})$ and allowing the higher one $(960 \mathrm{MHz})$.

It can be seen that for the chosen values, the D-CRLH lines are $50 \Omega$ balanced transmission lines at their corresponding frequencies so the equations in the design procedure are satisfied. The additional conventional transmission lines are chosen so that the input $T$ is matched at both frequencies. For this diplexer for separating $380 \mathrm{MHz}$ from $960 \mathrm{MHz}$, the value for the factor $k(N)$ is 2.01 . The conventional transmission lines allow joining the two branches and soldering the SMA connectors and, at the same time they are optimised to achieve the lowest return losses. The proposed circuit has been implemented on an Arlon600 substrate with a relative permittivity of 6 , height of $0.6 \mathrm{~mm}$. The photograph of the circuit is shown in Fig. 6.

Finally, the measurements show an excellent agreement with the simulations. In addition, the insertion losses for this diplexer are lower than $0.4 \mathrm{~dB}$ at both frequency bands. The return losses are lower than $-22 \mathrm{~dB}$ at each frequency band and at any diplexer port. Lastly, the isolation between the two output ports is nearly $40 \mathrm{~dB}$ at both working frequencies. In addition, the compactness of 
Table 1 Information on chip component

\begin{tabular}{|c|c|c|c|c|}
\hline & \multicolumn{5}{|c|}{$k(N)=2.01$} \\
\hline & Designed & Commercial & Self-resonance frequency, GHz & Tolerance, \% \\
\hline$L_{2 \mathrm{~L}}$ & $4.12 \mathrm{nH}$ & $08 \mathrm{HQ3N9}$ & 9 & \pm 5 \\
\hline$C_{2 \mathrm{~L}}$ & $1.65 \mathrm{pF}$ & GRM0222C1C1R7BDO & 20 & \pm 5 \\
\hline$L_{2 \mathrm{R}}$ & $16.66 \mathrm{nH}$ & $08 \mathrm{HQ} 16 \mathrm{~N}$ & 5 & \pm 5 \\
\hline$C_{2 \mathrm{R}}$ & $6.66 \mathrm{pF}$ & GRM0222C1C6R6BDO & 16 & \pm 5 \\
\hline$L_{3 \mathrm{~L}}$ & $10.42 \mathrm{nH}$ & $08 \mathrm{HQ} 10 \mathrm{~N}$ & 6 & \pm 5 \\
\hline$C_{3 \mathrm{~L}}$ & $4.17 \mathrm{pF}$ & GRM0222C1C4R3BD0 & 18 & \pm 5 \\
\hline$L_{3 \mathrm{R}}$ & $42.13 \mathrm{nH}$ & $08 \mathrm{HQ42N}$ & 2.5 & \pm 5 \\
\hline$C_{3 \mathrm{R}}$ & $16.66 \mathrm{pF}$ & GRM0222C1C16R0BD0 & 16 & \pm 5 \\
\hline
\end{tabular}
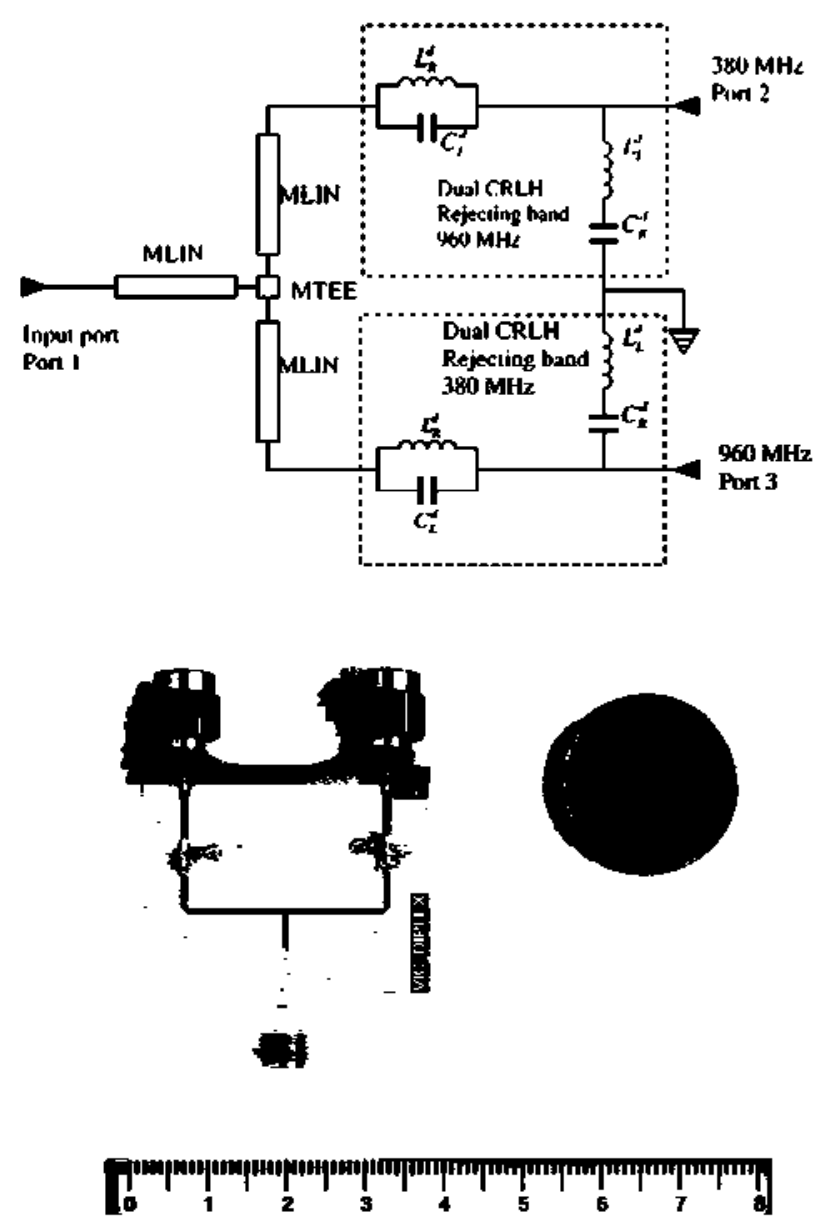

Figure 6 Schematic of the proposed design with one $D$-CRLH cell and photo of the proposed diplexer for separating $380 \mathrm{MHz}$ from $960 \mathrm{MHz}$

the circuit is quite good and miniaturisation has been achieved for a diplexer circuit working in the ultra high frequency (UHF) bands. The results can be seen from Figs. 7-9.

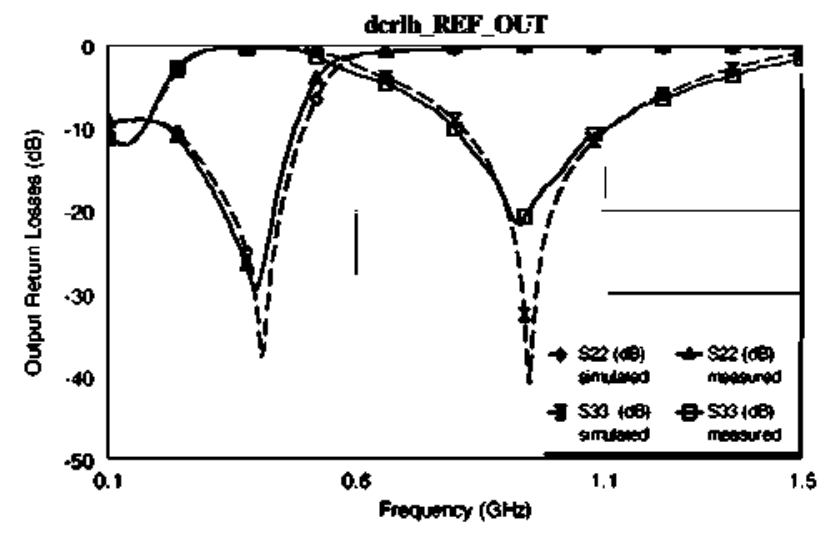

Figure 7 Simulated and measured output return losses for the D-CRLH GSM-TETRA diplexer at the two output ports (2 and 3)

\subsection{Diplexer for near frequencies}

A second diplexer for separating quite near frequencies has been manufactured and measured. The advantage of using D-CRLH technology allows us to obtain the corresponding working frequencies closer. This has not been achieved in other previous works for diplexing circuits (i.e. [8-11]). The selected bands are the corresponding to GSM and GALILEO, just to have miniaturisation and achieve lumped components where its resonance frequency is far away from the working frequency. For this diplexer for separating 1 and $1.2 \mathrm{GHz}$, the value for the factor $k(N)$ is 0.36 . Once again, following the design considerations in $(12)-(17)$, the associated component values are shown in Table 2. From the values in Table 2, it can be inferred that the balanced condition is satisfied. This table shows the designed values and the commercial chosen. In addition, the tolerance and the self-resonance frequency of each component are also shown to see the circuit sensitivity. Sub-index two corresponds to the branch rejecting the higher frequency $(1.2 \mathrm{GHz})$ and allowing the lower on $(1 \mathrm{GHz})$, while sub-index three corresponds to the branch 


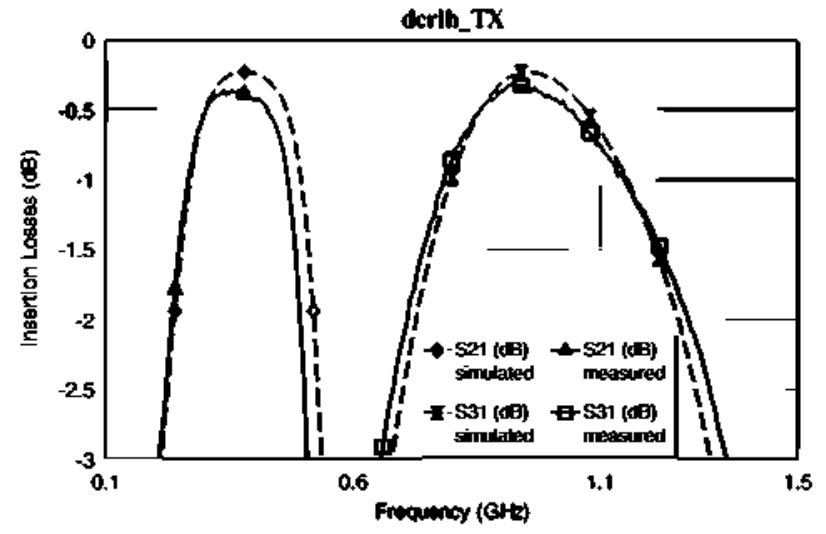

Figure 8 Detailed of the simulated and measured insertion losses for the D-CRLH GSM-TETRA diplexer

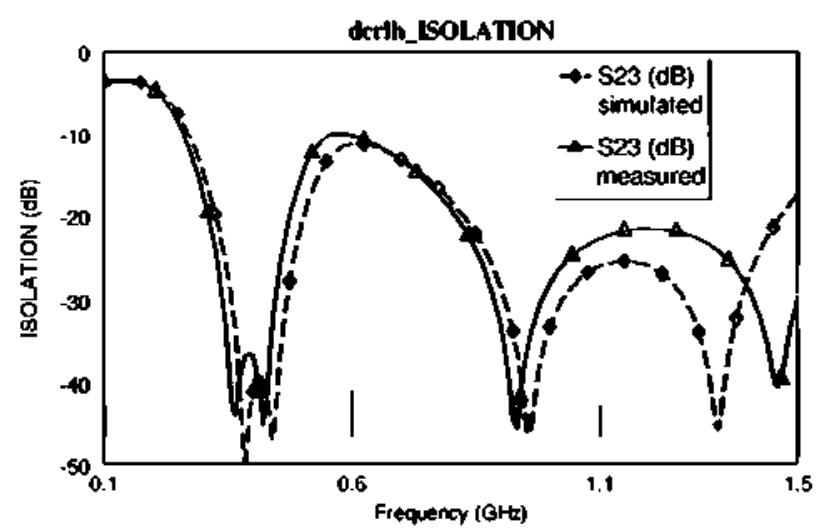

Figure 9 Simulated and measured isolation between output ports for the D-CRLH GSM-TETRA diplexer

rejecting the lower frequency $(1 \mathrm{GHz})$ and allowing the higher one $(1.2 \mathrm{GHz})$.

As in the previous case, it can be seen that for the chosen values the D-CRLH lines are $50 \Omega$ balanced transmission lines at their corresponding frequencies so the equations in the design procedure are satisfied. The additional
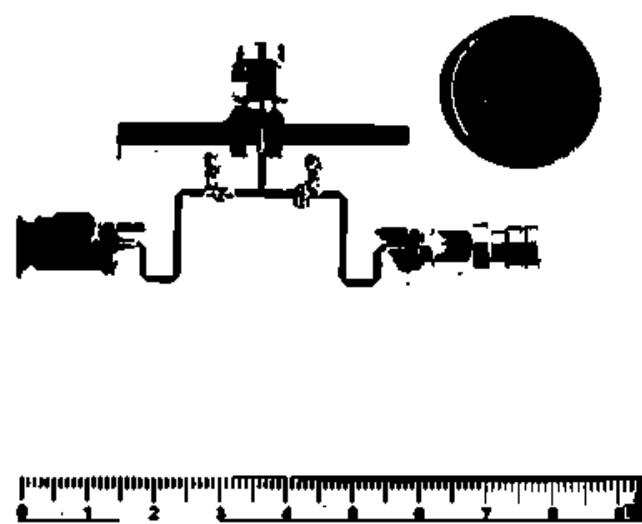

Figure 10 Photo of the proposed diplexer for separating frequencies in the GSM-GALILEO bands

conventional transmission lines are chosen so that the input $T$ is matched at both frequencies. The proposed circuit has been implemented on an Arlon 600 substrate with a relative permittivity of 6 , height of $0.6 \mathrm{~mm}$. The photograph of the circuit is shown in Fig. 10. Once again the output lines have been optimised to achieve the optimum return losses.

Finally, the results show good agreement with the simulations. However, as it will be seen in the next subsection the tolerance components and the nearby frequencies do not allow such a good performance as in the first example. The insertion losses for this diplexer for close frequencies are lower than $0.8 \mathrm{~dB}$ at the first frequency and $1.6 \mathrm{~dB}$ at the second frequency. The return losses at the output ports are around $-12 \mathrm{~dB}$ at the higher frequency and lower than $-17 \mathrm{~dB}$. Lastly, as the isolation between the two output ports is larger than $32 \mathrm{~dB}$ at both working frequencies there would not be any problem with potential interferences from port 2 to port 3. The results can be seen from Figs. 11-13.

\subsection{Sensitivity analysis}

It is clear that pure left-handed transmission lines are not available since there is always parasitic series inductance in

Table 2 Information on chip component for the second diplexer

\begin{tabular}{|l|c|c|c|c|}
\hline & Designed & Commercial & Self-resonance frequency, GHz & Tolerance, $\%$ \\
\hline$L_{2 \mathrm{~L}}$ & $25.2 \mathrm{nH}$ & $08 \mathrm{HQ25N}$ & 3 & \pm 5 \\
\hline$C_{2 \mathrm{~L}}$ & $10 \mathrm{pF}$ & GRM0222C1C10RBD0 & 14 & \pm 5 \\
\hline$L_{2 \mathrm{R}}$ & $1.75 \mathrm{nH}$ & $08 \mathrm{HQ} 1 \mathrm{~N} 8$ & 12.4 & \pm 5 \\
\hline$C_{2 \mathrm{R}}$ & $0.7 \mathrm{pF}$ & GRM0222C1CR7BD0 & 24 & \pm 5 \\
\hline$L_{3 \mathrm{~L}}$ & $30.03 \mathrm{nH}$ & $08 \mathrm{HQ30N}$ & 2.7 & \pm 5 \\
\hline$C_{3 \mathrm{~L}}$ & $12.01 \mathrm{pF}$ & GRM0222C1C12RBD0 & 12 & \pm 5 \\
\hline$L_{3 \mathrm{R}}$ & $2.11 \mathrm{nH}$ & $08 \mathrm{HQ2N} 2$ & 11.8 & \pm 5 \\
\hline$C_{3 \mathrm{R}}$ & $0.84 \mathrm{pF}$ & GRM0222C1CR8BD0 & 23 & \pm 5 \\
\hline
\end{tabular}




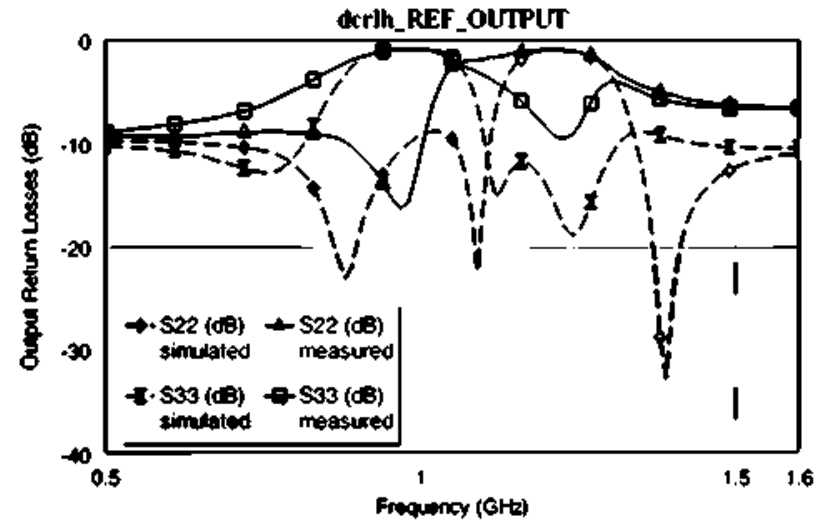

Figure 11 Simulated and measured output return losses for the D-CRLH GSM-GALILEO diplexer at the two output ports

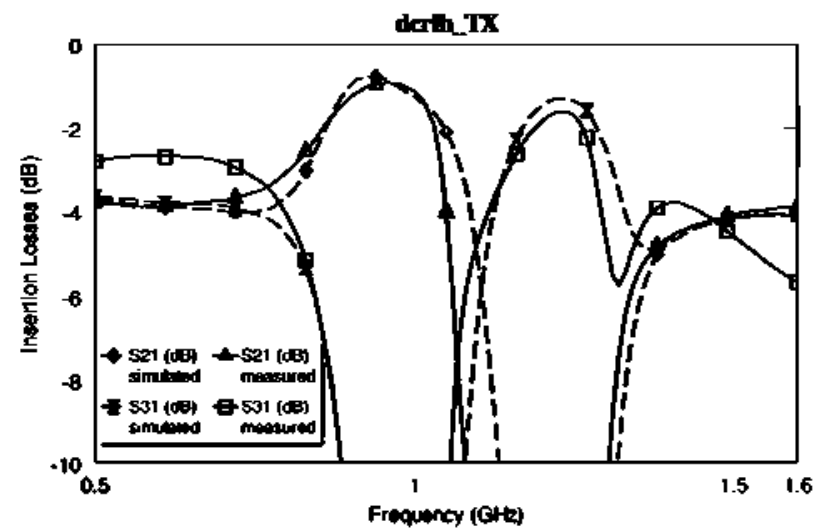

Figure 12 Detailed of the simulated and measured insertion losses for the D-CRLH GSM-GALILEO diplexer

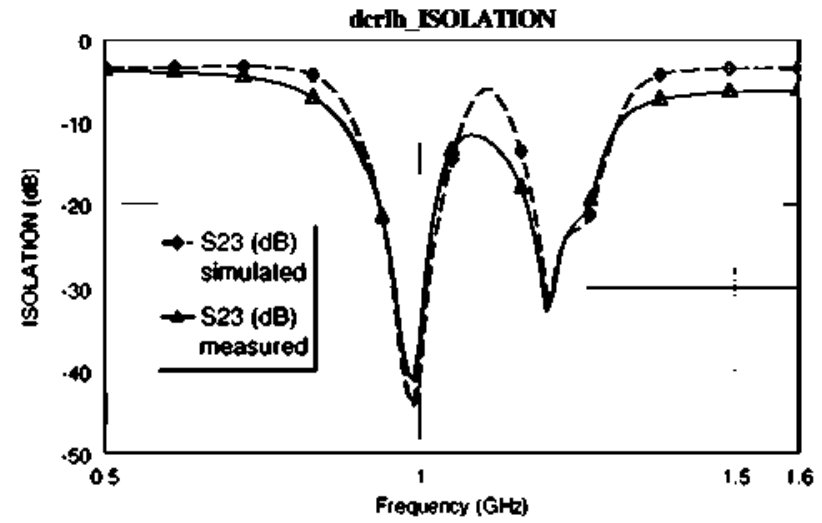

Figure 13 Simulated and measured isolation between output ports for the D-CRLH GSM-GALJLEO diplexer

the left-hand capacitance and parasitic-shunted capacitance in the left-handed inductor. Although that is true, the control on those parasitic elements is very hard. For that reason, when making metamaterial transmission lines, CRLH or D-CRLH lines have been used instead of pure LH ones. However, parasitic effects are always present. These effects can be classified into two groups: effects due

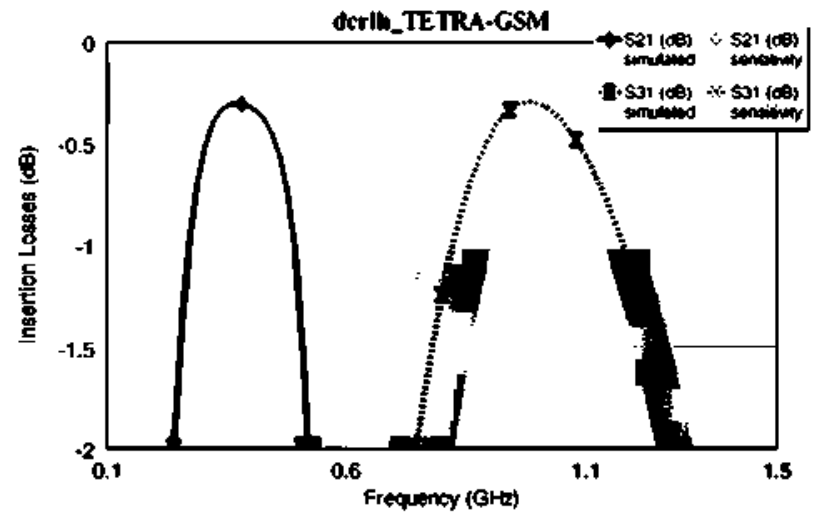

Figure 14 Sensitivity analysis for the insertion losses for the D-CRLH TETRA-GSM diplexer for a component variation of $5 \%$

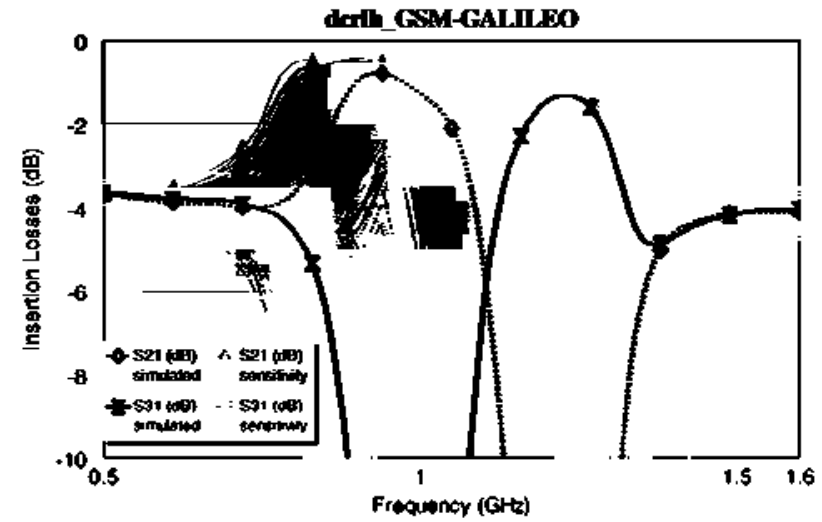

Figure 15 Sensitivity analysis for the insertion losses for the D-CRLH GSM-GALILEO diplexer for a component variation of $5 \%$

to the lumped-element tolerance that makes that the actual value differs from the nominal one and effects due to the presence of a parasitic inductance in the capacitors or a parasitic capacitance in the inductors.

In order to quantify the tolerance effect, a sensitivity analysis has been realised to show what the effect of these parasitic elements is and see whether the performance of the circuit is acceptable or not. However, this sensitivity analysis concerns the tolerance of the elements but not the effect of opposite parasitic elements. The effect of this is controlled by the fact that the chosen lumped elements present a very high self-resonance frequency, which indicates that the performance of the chosen lumped element is the one indicated by its nominal value.

For this sensitivity analysis, a tolerance for capacitors and inductors around their nominal value of about $5 \%$ has been considered. The statistical distribution for the components values has been taken as Gaussian and the number of repetitions is 2000 . The sensitivity analysis has been realised for both examples. It has been shown that the first 
prototype is very robust in front of the tolerance components. The specifications for the second prototype are much harder and, then, the sensitivity is lower. The sensitivity analyses for the insertion losses for both designs are finally shown in Figs. 14 and 15.

\section{Conclusion}

D-CRLH transmission lines have been shown as a good solution for the design of circuits that are alternatively used for rejecting or allowing different frequency bands. Then, a D-CRLH diplexer-circuit based on D-CRLH transmission lines has been proposed. A general design procedure for implementing arbitrary-frequency compact diplexers has been proposed. As the D-CRLH transmission lines do not present a frequency periodic performance, the proposed diplexer can be designed for every given frequency ratio $f_{02} / f_{03}$. Two first-order prototypes, one for TETRA-GSM and other for GSM-GALILEO, have been designed and manufactured. The results have shown the validity of the proposed design procedure for arbitrary frequencies.

\section{Acknowledgments}

This work was partially supported by grants from the Spanish MEC under projects TEC2006-13248-C04-04/TCM and TEC2009-14525-C02-01 and under CONSOLIDER CSD2008-0068.

\section{References}

[1] Matthaei G.l., Young L., Jones e.t.: 'Microwave filters impedance matching networks and coupling structure' (M.A. Artech House, 1964)

[2] WU K.L., MENG W.: 'A direct synthesis approach for microwave filters with a complex load and its applications to direct diplexer design', IEEE Trans. Microw. Theory Tech., 2007, 55, (5), pp. 1010-1017

[3] $x \cup$ w.a., HOM.H., HSU C.G.: 'UMTS diplexer design using dual-mode stripline ring resonators', Electron. Lett., 2007, 43, (13), pp. 721-722

[4] FRITZ M., WIESBECK W: 'A diplexer based on transmission lines, implemented in LTCC', IEEE Trans. Adv. Packag., 2006, 29, (3), pp. 427-432

[5] TANG C.W., You 5.F.: 'Design methodologies of LTCC bandpass filters, diplexer and triplexer transmission zeros',
IEEE Trans. Microw. Theory Tech., 2006, 54, (2), pp. $717-723$

[6] SANADA A., CALOZ C., ITOH T.: 'Characteristics of the composite right/left-handed transmission lines', IEEE Microw. Wirel. Compon. Lett., 2004, 14, (2), pp. 68-70

[7] CALOZ C., ITOH T.: 'Electromagnetic metamaterial transmission line theory and microwave applications' (Wiley, 2005)

[8] BONACHE J., GIL I., GARCIÁ-gARCIA J,, MARTíN F.: 'Complementary splits ring resonators for microstrip diplexer design', Electron. Lett., 2005, 41, (14), pp. 810-811

[9] HORII Y., CALOZC., ITOH T.: 'Super-compact multilayered Lefthanded transmission line and diplexer application', IEEE Trans. Microw. Theory Tech, 2005, 53, (4), pp. 1527-1534

[10] WONG J.K.H., BALMAIN K.G., ELEfTHERIADES G.V.: 'A diplexer based on the spatial filtering property of planar anisotropic transmission-line metamaterials'. Proc. IMS MTT, 2006

[11] CASTRO-GALAN D., GARCIA-MUÑOZ L.E., SEgOVIA-VARGAS D., GONZÁLEZ-POSADAS V.: 'Diversity monopulse antenna based on a dual frequency and dual mode CRLH rat-race coupler', Prog. Electromagn. Res., 2009, 14, pp. 87-106

[12] TONG W., NGUYEN H.V, HU Z, CALOZ C.l.: 'Dual composite right/left handed (D-CRLH) transmission line in GaAs MMIC technology'. Proc. IMS MTT, 2007

[13] CALOz C.: 'Dual composite right/left-handed (D-CRLH) transmission line metamaterial', IEEE Microw. Wirel. Compon. Lett., 2006, 16, (11), pp. 585-587

[14] HU X., ZHANG P.: 'A novel dual-band balun based on the dual structure of composite right/left handed transmission line'. Proc. Biophotonics, Nanophotonics and Metamaterials, Rome, October 2006, pp. 529-532

[15] HU X., ZHANG P., HE S.: 'Dual structure of composite right/ left handed transmission line', J. Zhejiang Univ. Sci. A, 2006, 1, pp. 529-532

[16] HERRAIZ-MARTINEZ F.J., GONZÁLZZ-POSADAS V., IN̈IGO-VILLACORTA F., SEGOVIA-VARGAS D.: 'Low-cost approach based on an eigenfrequency method to obtain the dispersion diagram in CRLH structures', IEEE Microw. Wirel. Compon. Lett., 2007, 17, (1), pp. 13-15 